\title{
Allopurinol reduces left ventricular hypertrophy
}

In patients with chronic kidney disease (CKD), treatment with allopurinol-a xanthine oxidase inhibitor-can improve endothelial function and reduce left ventricular hypertrophy, according to a recent paper.

Cardiovascular disease is the primary cause of death in patients with CKD, a fact that is partly attributable to the high prevalence of left ventricular hypertrophy in these patients. "Conventionally, left ventricular hypertrophy regression is achieved by reducing blood pressure by means of antihypertensives," states lead author Michelle Kao. However, in patients with CKD, methods of reducing left ventricular hypertrophy that do not rely on lowering blood pressure are required, as the use of medications that reduce blood pressure is often contraindicated in these patients. Allopurinol, which does not primarily act by reducing blood pressure, is known to improve endothelial and vascular function in patients who do not have CKD. In addition, two animal models have demonstrated that allopurinol can regress left ventricular mass. Kao and colleagues decided to evaluate whether these effects also occur in patients with CKD. The researchers also assessed the effect of allopurinol on endothelial dysfunction, which is a surrogate marker of vascular health.

The researchers randomly allocated 53 patients with stage 3 CKD and left

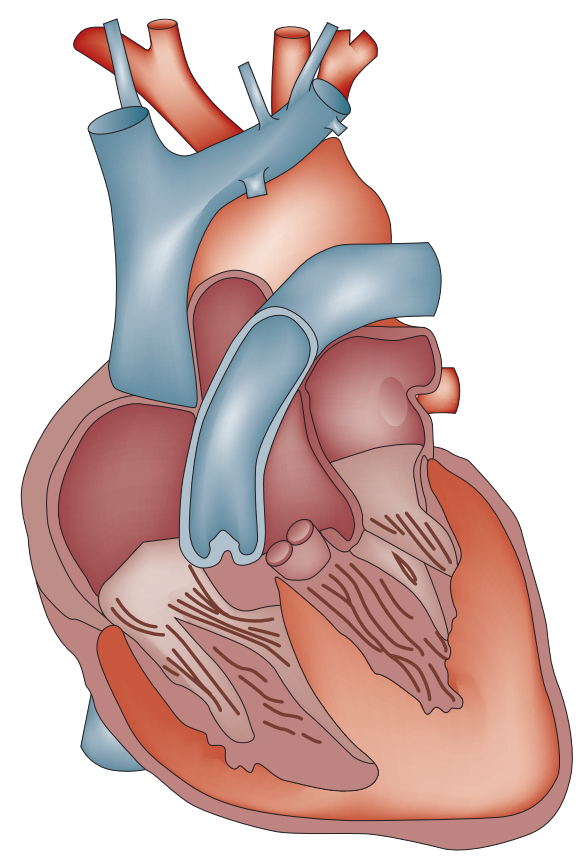

ventricular hypertrophy to receive either allopurinol or placebo for 9 months. The dosage of allopurinol was initially $100 \mathrm{mg} /$ day and was increased to $300 \mathrm{mg} /$ day after 2 weeks if there were no adverse effects. The left ventricular mass index was measured with cardiac MRI, endothelial function was assessed using flowmediated dilation of the brachial artery and central arterial stiffness was assessed with pulse-wave analysis.

Compared with patients who received placebo, those who received allopurinol had a reduced left ventricular mass index as well as improved endothelial function and reduced arterial stiffness after 9 months of treatment. Allopurinol at $300 \mathrm{mg} /$ day was well tolerated, and no patient experienced a notable decline in renal function. However, $7 \%$ of patients who received allopurinol developed a rash, which the authors note could prevent its widespread use.

The authors speculate that the vascular effects of allopurinol could underlie its action on left ventricular hypertrophy. "Allopurinol regressed left ventricular mass because of decreased left ventricular afterload as a consequence of better vascular compliance and reduced arterial wave reflection," explains Kao.

The study by Kao and colleagues is one of the first to show that allopurinol is beneficial in patients with CKD. "These data suggest that many further studies would be worthwhile; for example, regression studies with higher doses of allopurinol, longer time frames and possibly even studies in patients with advanced stages of CKD," states Kao.

\section{Claire Greenhill}

Original article Kao, M. P. et al. Allopurinol benefits left ventricular mass and endothelial dysfunction in chronic kidney disease. J. Am. Soc. Nephrol. 22, 1382-1389 (2011) 